\title{
Building momentum for systems and synthetic biology in India
}

\author{
Remya Krishnan · Lijo Anto Manjaly-Antony • \\ Pawan K. Dhar
}

Received: 29 January 2011/Accepted: 3 February 2011/Published online: 23 February 2011

(C) Springer Science+Business Media B.V. 2011

\begin{abstract}
Biological systems are inherently noisy. Predicting the outcome of a perturbation is extremely challenging. Traditional reductionist approach of describing properties of parts, vis-a-vis higher level behaviour has led to enormous understanding of fundamental molecular level biology. This approach typically consists of converting genes into junk (knock-down) and garbage (knock-out) and observe how a system responds. To enable broader understanding of biological dynamics, an integrated computational and experimental strategy was formally proposed in mid 1990s leading to the re-emergence of Systems Biology. However, soon it became clear that natural systems were far more complex than expected. A new strategy to address biological complexity was proposed at MIT (Massachusetts Institute of Technology) in June 2004, when the first meeting of synthetic biology was held. Though the term 'synthetic biology' was proposed during 1970s (Szybalski in Control of gene expression, Plenum Press, New York, 1974), the usage of the original concept found an experimental proof in 2000 with the demonstration of a three-gene circuit called repressilator (Elowitz and Leibler in Nature, 403:335-338, 2000). This encouraged people to think of forward engineering biology from a set of well described parts.
\end{abstract}

Keywords Systems biology $\cdot$ Synthetic biology $\cdot$ India

R. Krishnan · L. A. Manjaly-Antony

Centre for Bioinformatics, University of Kerala, Kerala, India

R. Krishnan · P. K. Dhar $(\bowtie)$

Centre for Systems and Synthetic Biology, University of Kerala,

Trivandrum, Kerala 695 581, India

e-mail: pawan@cssb.res.in
To identify the emerging synthetic biology community in India, the first synthetic biology symposium called Biodesign India 1.0 was held at the University of Kerala from October 7-9, 2010. The event featured 18 speakers, 50 poster presentations from different parts of the country. Talks ranged from fundamental biology to technology, from bioethics to biosafety and biosecurity issues.

In her plenary talk, Manju Bansal from Indian Institute of Sciences, Bangalore asked whether synthetic biology research was leading towards the emergence of synthetic life? Her work involves rational design of novel proteins with pre-defined structure and function, including use of unnatural amino acids. She observed that adapting organisms towards producing useful molecules, from drugs to bio-fuels, was a realistic goal worthy of pursuit. Her work over the past several years has led to the identification of key parameters essential for the construction of a promoter. These parameters include curvature, bendability and stability. By using existing experimental data and computationally applying three key parameters to whole genome sequences, promoter regions were predicted by studying differences between known promoters and non-promoter sequences. Briefly, these terms describe the inherent capability of DNA to bend around a given three dimensional surface, the extent to which an incoming protein can bend DNA molecule and maximize contact points, and the stability of DNA double helix and DNA-protein interaction. Manju Bansal showed that lower stability of DNA duplex, high curvature and less bendability are key signatures of promoter sequences. Her team has developed a tool called PromPredict that uses a set of free energy threshold values for genomic DNA with varying GC content. The tool shows 99 and $95 \%$ sensitivity on E. coli and B. subtilis genomic data and $\sim 60 \%$ precision-the highest values obtained so far. Her team's work also enables targeted 
modification of promoter sequences and engineering new ones.

Ramakrishna Ramaswamy from Jawaharlal Nehru University, Delhi shared his recent findings on the effects of stochasticity on the synchrony and multi-stability of regulatory networks. The negative auto-regulation of gene expression generated oscillations in the network. The stochastic model showed that miRNA expression reduced the amplitude and frequency of oscillations thereby regulating the dynamics of protein production.

Venkatesh Kareenhalli from Indian Institute of Technology Mumbai, described how his lab designed and characterized multiple feedback loops using synthetic gene constructs. Computational models of feedback loops were experimentally validated. One interesting outcome of his study was the observation that multiple feedback loops offered distinct advantage in terms of speed of response, robustness and attenuation of noise.

Guhan Jayaraman from the Indian Institute of Technology, Madras talked about combining systems and synthetic biology approaches to study how chaperons interacted with recombinant proteins to increase the protein output and quality of yield. Chaperones were co-expressed with recombinant proteins to modulate solubilization and re-folding. DnaK-DnaJ-GrpE chaperones were found to be overproduced with cytoplasmic recombinant proteins tending to form inclusion bodies. The key message was the use of chaperon cocktails to significantly increase the yield of protein production.

Narahari Sastry from the Indian Institute of Chemical Technology, Hyderabad shared his work on molecular similarity, diversity and virtual screening. He observed that an important filter in virtual screening was the chemical diversity of compounds e.g., R-Salbutamol was found to be effective in asthma patients while L-Salbutamol had a detrimental effect on these patients. The molecular structure representation could be done using connection table, adjacency matrix, feature tree, hashed fingerprints, pharmacophore mapping, correlation vector, graph theory etc. The similarity between compounds could be determined by equivalence classes, numerical measures, distance measures. He illustrated the use of such approaches in synthetic biology based on ligand and structure based protocols.

Vijaya Gopal from the Centre for Cellular and Molecular Biology, Hyderabad showed her work on designing multi-domain fusion proteins for cell-specific DNA targeting. She is pursuing the goal of transfer and targeted integration of therapeutic nucleic acids into cells, which critically depends upon design and construction of efficient carriers. Dr.Vijaya started by describing computational studies for predicting structure-function of designed peptides apriori leading to design of recombinant peptide chimeras in vitro. Interestingly, chimeras designed for cell- targeting moieties retain their functional properties. She described potential applications of her work towards designing targeted in vivo delivery of siRNA.

B. Padmanabhan, from Aptuit Laurus, Genome Valley, Hyderabad demonstrated how his team designed a novel nucleoside analog as a potent inhibitor of the NAD+ Dependent Deacetylase, SIRT2. Sirtuins are unique NAD dependent histone deacetylases (HDACs) that deacetylate their target proteins. Apart from regulating physiological functions, they are found to have relevance in Cancer, Obesity, Diabetes and in life span. Genetic algorithm was used to identify approximately 100 compounds that contain SIRT2 protein. Among these 100 compounds, SIRT2 was found to be inhibited with a nucleoside analogue (AL-1). The inhibitory process was also carried out for SIRT1 against this compound, and it exhibited less inhibition activity against SIRT1 compared to SIRT2.

D. Sundar from the Department of Biochemical Engineering and Biotechnology, Indian Institute of Technology, Delhi described his work on designing proteins for application in human therapeutics. His study involved the use of Zinc finger proteins- a 24-30 amino acid long, DNA binding protein with two anti-parallel beta strands and a alpha helix where two cysteine and two histidine ligands bind a zinc ion. By stringing several zinc fingers (each can recognize 3 bp of DNA) together he demonstrated the feasibility of creating multiple zinc finger proteins for recognizing unique DNA sequence in any genome with high specificity and affinity. This has tremendous applications towards modular protein engineering leading to health applications.

Vishwesh Kulkarni from Indian Institute of Technology, Bombay gave an interesting perspective on building binary counting with chemical reactions. The 3-bit counter and an FIR filter realized were using biomolecular reactions. Error was of the order of exp $(-3)$. He proposed that the 3-bit counter could be extended to N-bit counter to build AND, OR gates. He presented stochastic simulation results that validated their construct and outlined how the DNA-based computation via strand displacement could be a promising platform for implementing this construct. Potential applications of this counter include biosensing and drug delivery. He outlined steps to build biomolecular computer from DNA, in the long run.

Archana Chugh from the School of Biological Sciences, Indian Institute of Technology, Delhi described legal and socio-ethical implications of synthetic biology. She expressed concern that the public awareness and consideration of health related issues arising from this new field were strikingly low in India. Dr. Archana raised potential social, legal, ethical, environmental and safety issues arising from synthetic biology. She made recommendations for the controlled practice of this new field that presented itself with a strong potential for dual use. 
Yashas Shetty from the Centre for Media Arts, Bangalore demonstrated artistic impression of synthetic biology. He shared the work of Symbiotica on tissue engineering who deal with developing 'ethical' meat, design artificial leather jacket without slaughtering animals, and showed a new form of biological art where colored pens controlled by a mouse brain generated electrical activity of neurons resulting in interesting patterns on paper. At the Srishti School of Art and Design his students have successfully constructed low cost innovation products e.g., microscope from Web cam, a low speed centrifuge from egg beater and low cost incubator designed from cardboard.

Indira Ghosh from Jawahar lal Nehru University, Delhi described her work on developing a targeted drug for infectious diseases. The Mycobacterium TB model of her lab reproduced the observation that deletion of either of the two Iso-Citrate lyase (ICL) genes had little effect on the bacterial growth in macrophages, but deletion of both genes lead to the elimination of the bacilli and sustained the inhibition of ICLs by 3-NP (3 Nitro propionic acid). She said that intracellular domain kinase needed for glyoxylate bypass flux in persistent bacteria was a potential drug target. Computational modeling and in silico inhibiton of the enzymes showed that 4 out of 7 enzymes of MEP (2-C-methylerythritol 4-phosphate) pathway could be potential drug targets.

P. Gautham from Anna University, Chennai, described his work of using machine learning algorithms to predict unique metal ion binding sites of proteins. His machine learning algorithms predict a global fingerprint of metalion binding proteins that could not be captured through sequence analysis. He demonstrated how algorithm could be used to synthetically modify proteins to include a motif specific to a metal or present a global fingerprint making it a putative metal-ion binding protein.

H. V. Thulasiram of the National Chemical Laboratory, Pune gave an insight on the biosynthesis of isoprenoids using micro-organisms as "models for mammalian metabolism". Isoprenoid compounds are deeply integrated into crucial metabolic functions in all cells. Santalols (constitutes about $90 \%$ of the Heartwood oil of Santalum album) and their derivatives show considerable activity against the antibiotic (metronidazole, clarithromycin) resistant strain of H. pylori. His work shows that santalane synthase converts FPP (Farnesyl pyrophosphate) synthesized by mevalonate pathway to santalenes. Hydroxylation of these santalenes forms santalols which can be done by microbial transformation. Using recombinant DNA technology methods, his lab recently demonstrated an increase the production of santalols using synthetic biology approach.

Nagasuma Chandra from Indian Institute of Science, Bangalore presented systems approach to study and understand the function of biological systems, particularly, metabolism of Mycobacterium tuberculosis (Mtb). She discussed multiple paradigm shifts that the drug discovery field was witnessing. Her talk focused on metabolic modeling in $M t b$. She began by describing constraint based model of mycolic acid pathway (MAP), which also happened to be the target of known drugs such as isoniazid and Ethambutol. Using flux balance analysis, a method for analyzing the theoretical capabilities of metabolic system, she showed how the pathway could be studied how insights on various aspects such as essentiality and critical reactions required for growth of an organism could be obtained. She presented a novel drug discovery target developed in her laboratory, that integrates systems level studies, protein interactome, flux balance analysis of the reactome, with sequence analysis and structural assessment of the targetability.

Manish Gupta from Dhirubhai Ambani Institute of Information and Communication Technology describe a fascinating and new area of DNA self assembly. Selfassembly is a process by which supramolecular species are created from their elementary building blocks. He stressed on questions like : is it possible to self assemble biomolecules such as DNA at our wish? He described how Erik Winfree of Caltech showed that DNA self assembly is Turing universal. This means that rather than using trial and error we could systematically generate the desired structure and also perform computation by DNA selfassembly. He further described how in 2006, Paul Rothemund at Caltech created DNA smileys, map of North America using a kind of DNA stapler on viruses. This paved the way to a field known as DNA origami. He predicted that in future self-assembly will become an important discipline by combining the fields of bio-molecular computation, nano-technology, medicine and synthetic biology. In his talk he provide an overview of this interdisciplinary area of research and described his work towards error-control in DNA self assembly.

Vibin Ramakrishnan from Rajiv Gandhi Centre for Biotechnology, Kerala described his strategy to develop novel proteins using non natural amino acids. He asked several interesting questions: (1) Is there a way to produce newer amino acids that can participate in protein formation? (2) Is it possible to have D-Chiral amino acids in proteins? His study involved the use of Automated Repetitive-Simulated Annealing Molecular Dynamics (AR-SAMD) method to search for novel folds. One stereoisomer was subjected to racemization to obtain 109 stereo-isomers. Folding process was carried out to obtain 109 folds which gave rise to a primordial soup of structures. Finally all the folds were analyzed using AR-SAMD to obtain the best fold. Studies also showed that proteins of several different folds and thus proteins with a variety of different functions could be formed if it had D-amino acids. He predicted that the vast and potentially unexplored terrain in the protein universe 
was likely to be the face and future of artificial biomaterials in the future.

Pawan K. Dhar of the Centre for Systems and Synthetic Biology, University of Kerala described his novel approach of making proteins from intergenic regions of E.coli. His team was successful in expressing proteins from noncoding DNA and observed interesting structural and phenotypic correlations. Further, he described the computational synthetic biology work of developing Genome Designer-a novel bioCAD platform for biological engineering.

In addition to the full length talks, a series of short talks were presented. M. V. Jagannadham of Centre for Cellular and Molecular Biology Hyderabad described his work of identifying more than 1200 proteins of Antartic bacterium Pseudomonas syringe, including 120 outer membrane proteins. Navneet Rai of NCBS (National Centre for Biological Sciences) Bangalore described the history, outcome and limitations of the iGEM competition. He also walked through a quick description of the Registry of Standard Biological Parts and highlighted the need of a similar competition based on synthetic biology in India. Pushkar Malakar of IIT Bombay described how he designed synthetic constructs in E. coli to mimic multiple feedback loops. Dipesh D. Jadhav of National Chemical Labs, Pune presented findings on lipases from several microorganisms which have been used to achieve chiral resolution of some of the cyclic and acyclic alcohols and fragrance compounds. Ashwini Bhat from the Indian Institute of Sciences, Bangalore described metabolic adjustments in Mycobacterium tuberculosis upon exposure to isoniazid (INH), a front-line drug in tuberculosis treatment. Her talk provided in depth insight for target identification and combination therapy.

Samit Watve from IISER (Indian Institute of Science Education and Research), Pune described his work on building synthetic devices in plants using Agrobacteriummediated plant transformation. Further, he outlined tremendous advantages and potential challenges in the area of plant synthetic biology. Girinath Pillai from Centre for Bioinformatics gave a $2 \mathrm{~h}$ tutorial on drug designing. Towards the end of the 2 day event, there was a $2 \mathrm{~h}$ open house discussion on synthetic biology which was the highlight of the event. Of a number of questions that were raised, some of them received greater attention e.g., what is synthetic biology, what are its success stories? What are the comparative merits of bio-brick vs non-bio-brick initiatives, emerging business opportunities, funding, ethics and safety issues. Finally, a broad outline of course curriculum in synthetic biology and the future employment opportunities in synthetic biology, were discussed. It was also opined unanimously that the Indian Government urgently needed to devise a new policy with immediate effect, covering biosafety and biosecurity issues emerging from synthetic biology research.

Acknowledgments Our warmest thanks to Dr. Achuthsankar S. Nair for his strong support and Bio Design India Team at the Centre for Systems and Synthetic Biology/Centre for Bioinformatics, University of Kerala covering the event. In particular, we would like to thank Anju Ratheesh, Merin Aldo, Aiswarya, Sheeba K. and Prathibha M. C. for their help in documentation process.

\section{References}

Elowitz MB, Leibler S (2000) A synthetic oscillatory network of transcriptional regulators. Nature 403:335-338

Szybalski W (1974) In vivo and in vitro initiation of transcription. p 405. In: Kohn A, Shatkay A (eds) Control of gene expression. pp 23-24, and Discussion pp. 404-405, Szybalski's concept of Synthetic Biology. pp 411-412, pp 415-417. Plenum Press, New York 